\title{
Instanton corrections to the quark form factor at large momentum transfer
}

\author{
Alexander E. Dorokhov* and Igor O. Cherednikov ${ }^{\dagger}$ \\ Bogoliubov Laboratory of Theoretical Physics, Joint Institute for Nuclear Research, 141980 Dubna, Russia
}

(Received 4 March 2003; published 20 June 2003)

\begin{abstract}
Within the Wilson integral formalism, we discuss the structure of nonperturbative corrections to the quark form factor at large momentum transfer analyzing the infrared renormalon and instanton effects. We show that the nonperturbative effects determine the initial value for the perturbative evolution of the quark form factor and attribute their general structure to the renormalon ambiguities of the perturbative series. It is demonstrated that the instanton contributions result in the finite renormalization of the next-to-leading perturbative result and numerically are characterized by a small factor reflecting the diluteness of the QCD vacuum within the instanton liquid model.
\end{abstract}

DOI: 10.1103/PhysRevD.67.114017

PACS number(s): 12.38.Lg, 11.10.Gh

\section{INTRODUCTION}

The various aspects of the instanton induced effects in high energy QCD processes had been addressed at the very beginning of the instanton era (see, e.g., Ref. [1]), and this study has been continued in later decades [2]. Recently, the interest in them has been revived [3-7], and the hope of the direct detection of the instanton induced effects has appeared [8]. One of the important questions in the description of hadronic exclusive processes is the behavior of the form factors in various energy domains. The present paper is devoted to the analysis of the corrections to the elastic quark form factor at large momentum transfer induced by the infrared (IR) renormalon [9] and instanton effects, treating the latter within the framework of the instanton liquid model of the QCD vacuum [10].

From the theoretical point of view, the form factor analysis requires a perturbative resummation procedure beyond the standard renormalization group techniques, since it exhibits a double-logarithmic behavior. In addition to this, the resummation techniques developed for this particular case can be applied to the study of many other processes which possesses the logarithmic enhancements near the kinematic boundaries. On the other hand, in addition to this obvious theoretical use, the computation of the quark form factor has important phenomenological applications. A similar resummation approach is also used in study of the near-forward quark-quark scattering and evaluation of the soft Pomeron properties where the nonleading logarithmic contributions are quite important [11]. The application of this formalism in heavy quark effective theory can also be useful [12]. The quark form factor itself enters into the various cross sections of high-energy processes [13]. In particular, this quantity finds the most straightforward phenomenological application in the total cross section of the Drell-Yan process in the deep inelastic scattering (DIS) scheme, which is proportional to the ratio of the timelike and spacelike form factors [14-16].

\footnotetext{
*Electronic address: dorokhov@thsun1.jinr.ru

${ }^{\dagger}$ Also at: Institute for Theoretical Problems of Microphysics, Moscow State University, 119899 Moscow, Russia; electronic address: igorch@thsun1.jinr.ru
}

In this case, the exponentiated quark form factor is expressed in terms of the evolution equation, and can be evaluated, in principle, to any order in perturbation theory. The analysis shows that this is the high-energy asymptotic behavior that is important, and all the logarithmic contributions must be taken into account equally, while the power corrections could be neglected [16]. The investigation of the electomagnetic quark form factors in moderate and low energy domains can shed light on the problem of scaling violation in DIS and the structure of constituent quarks [17].

The color singlet quark form factor is determined via the elastic scattering amplitude of a quark in electromagnetic field

$$
\mathcal{M}_{\mu}=F_{q}\left[\left(p_{1}-p_{2}\right)^{2}\right] \bar{u}\left(p_{1}\right) \gamma_{\mu} v\left(p_{2}\right),
$$

where $u\left(p_{1}\right), v\left(p_{2}\right)$ are the spinors of outgoing and incoming quarks. The kinematics of the process is described in terms of the scattering angle $\chi$ :

$$
\begin{aligned}
\cosh \chi & =\frac{\left(p_{1} p_{2}\right)}{m^{2}}=1+\frac{Q^{2}}{2 m^{2}}, \\
Q^{2} & =-\left(p_{2}-p_{1}\right)^{2}>0, \\
p_{1}^{2} & =p_{2}^{2}=m^{2} .
\end{aligned}
$$

It is known that the leading large- $Q^{2}$ asymptotics of the quark form factor is given by the exponentiation of the oneloop term [18]

$$
F_{q}^{(1)}\left(Q^{2}\right)=\exp \left(-\frac{\alpha_{s}}{4 \pi} C_{F} \ln ^{2} \frac{Q^{2}}{\lambda^{2}}\right)+O\left(\alpha_{s}^{n} \ln ^{2 n-1} \frac{Q^{2}}{\lambda^{2}}\right)
$$

where $\lambda$ is an IR cutoff parameter. In general, for a correct consideration of the nonleading asymptotic contributions one has to resum all perturbative [such as $O\left(\alpha_{s}^{n} \ln ^{2 n-1} Q^{2}\right)$, $O\left(\alpha_{s}^{n} \ln ^{2 n-2} Q^{2}\right)$, etc.] as well as nonperturbative terms. An effective framework for resummation of perturbative and nonperturbative contributions is provided by the Wilson integral approach [19]. Within this framework, the resummation of all logarithmic terms coming from the soft gluon 
subprocesses allows us to express the quark form factor (3), in terms of the vacuum average of the gauge invariant path ordered Wilson integral [20]

$$
W\left(C_{\chi}\right)=\frac{1}{N_{c}} \operatorname{Tr}\left\langle 0\left|\mathcal{P} \exp \left(i g \int_{C_{\chi}} d x_{\mu} \hat{A}_{\mu}(x)\right)\right| 0\right\rangle .
$$

In Eq. (4) the integration path corresponding to considering process goes along the closed contour $C_{\chi}$ : the angle (cusp) with infinite sides. The gauge field

$$
\hat{A}_{\mu}(x)=T^{a} A_{\mu}^{a}(x), \quad T^{a}=\frac{\lambda^{a}}{2},
$$

belongs to the Lie algebra of the gauge group $S U\left(N_{c}\right)$, while the Wilson loop operator $\mathcal{P} \mathrm{e}^{i g \int d x A(x)}$ lies in its fundamental representation.

In our recent paper [7], we applied the Wilson integral formalism to evaluation of the perturbative and nonperturbative contributions to the color singlet quark form factor at the low normalization point $\mu$ of order of the inverse instanton size within the instanton liquid model. In the present work, considering the renormalization group (RG) evolution equation we extend the analysis to the limit of large momentum transfers focusing on the asymptotic behavior. We show that the nonperturbative effects determine the initial value for the perturbative evolution, find their general structure by analyzing the renormalon ambiguities of the perturbative series, and establish the correspondence between them and the instanton induced contribution.

The paper is organized as follows. In Sec. II we reproduce the known results of the perturbative one-loop calculation, and derive the evolution equations taking into account the nonperturbative contribution as the initial value for perturbative evolution. In Sec. III, we study the consequences of the IR renormalon ambiguities of the perturbative series and show how the latter prescribes the form of the nonperturbative corrections to the asymptotic behavior of the form factor at large $Q^{2}$. In Sec. IV, these nonperturbative effects are estimated in the weak-field approximation within the instanton model of the QCD vacuum. Finally, the large- $Q^{2}$ behavior of the form factor is analyzed taking into account the leading perturbative, IR renormalon, and instanton induced contributions. The latter are found to be determined by small factor expressed via the parameters of the instanton liquid model.

\section{ANALYSIS OF THE PERTURBATIVE CONTRIBUTIONS TO THE WILSON INTEGRAL}

The Wilson integral (4) can be presented as a series

$$
\begin{aligned}
W\left(C_{\chi}\right)= & +\frac{1}{N_{c}}\langle 0| \sum_{n=2}(i g)^{n} \int_{C_{\chi}} \int_{C_{\chi}} \ldots \int_{C_{\chi}} d x_{\mu_{n}}^{n} \\
& \times d x_{\mu_{n-1}^{n-1}} \cdots d x_{\mu_{1}}^{1} \theta\left(x^{n}, x^{n-1}, \ldots, x^{1}\right) \\
& \times \operatorname{Tr}\left[\hat{A}_{\mu_{n}}\left(x^{n}\right) \hat{A}_{\mu_{n-1}}\left(x^{n-1}\right) \cdots \hat{A}_{\mu_{1}}\left(x^{1}\right)\right]|0\rangle,
\end{aligned}
$$

where the function $\theta(x)$ orders the color matrices along the integration contour. In the present work, we restrict ourselves with the study of the leading order (one loop-for the perturbative gauge field and weak-field limit for the instanton) terms of the expansion (6) which are given by the expression

$$
W^{(1)}\left(C_{\chi}\right)=-\frac{g^{2} C_{F}}{2} \int_{C_{\chi}} d x_{\mu} \int_{C_{\chi}} d y_{\nu} D_{\mu \nu}(x-y),
$$

where the gauge field propagator $D_{\mu \nu}(z)$ in $n$-dimensional space-time $(n=4-2 \varepsilon)$ can be presented in the form

$$
D_{\mu \nu}(z)=g_{\mu \nu} \partial_{z}^{2} \Delta_{1}\left(\varepsilon, z^{2}, \mu^{2} / \lambda^{2}\right)-\partial_{\mu} \partial_{\nu} \Delta_{2}\left(\varepsilon, z^{2}, \mu^{2} / \lambda^{2}\right) .
$$

The exponentiation theorem for non-Abelian path-ordered Wilson integrals $[21,22]$ allows us to express (to one-loop accuracy) the Wilson integral (4) as the exponentiated oneloop term of the series (6):

$$
W\left(C_{\chi}\right)=\exp \left[W^{(1)}\left(C_{\chi}\right)+O\left(\alpha_{s}^{2}\right)\right] .
$$

In general, the expression (7) contains ultraviolet (UV) and IR divergences, that can be multiplicatively renormalized in a consistent way [23]. In contrast to the previous paper [7], we use the dimensional regularization in order to work with UV singularities, and define the "gluon mass" $\lambda^{2}$ as the IR regulator and the parameter $\mu^{2}$ as the UV normalization point. The dimensionally regularized formula for the leading order (LO) term (7) can be written as [7]

$$
\begin{aligned}
& W^{(1)}\left(C_{\chi} ; \varepsilon, \mu^{2} / \lambda^{2}, \alpha_{s}\right) \\
& \quad=8 \pi \alpha_{s} C_{F} h(\chi)(1-\varepsilon) \Delta_{1}\left(\varepsilon, 0, \mu^{2} / \lambda^{2}\right),
\end{aligned}
$$

where $h(\chi)$ is the universal cusp factor,

$$
h(\chi)=\chi \operatorname{coth} \chi-1,
$$

and, in case of the perturbative field,

$$
\Delta_{1}\left(\varepsilon, 0, \mu^{2} / \lambda^{2}\right)=-\frac{1}{16 \pi^{2}}\left(4 \pi \frac{\mu^{2}}{\lambda^{2}}\right)^{\varepsilon} \frac{\Gamma(\varepsilon)}{1-\varepsilon} .
$$

The independence of the expression (10) of the function $\Delta_{2}$ is a direct consequence of the gauge invariance. Then, in the one-loop approximation

$$
\begin{aligned}
& W\left[C_{\chi} ; \varepsilon, \mu^{2} / \lambda^{2}, \alpha_{s}(\mu)\right] \\
& \quad=1-\frac{\alpha_{s}(\mu)}{2 \pi} C_{F} h(\chi)\left(\frac{1}{\varepsilon}-\gamma_{E}+\ln 4 \pi+\ln \frac{\mu^{2}}{\lambda^{2}}\right),
\end{aligned}
$$

and the cusp dependent renormalization constant [23], within the modified minimal subtraction scheme, reads

$$
Z_{\text {cusp }}\left[C_{\chi} ; \varepsilon, \alpha_{s}(\mu)\right]=1+\frac{\alpha_{s}(\mu)}{2 \pi} C_{F} h(\chi)\left(\frac{1}{\varepsilon}-\gamma_{E}+\ln 4 \pi\right) .
$$


The detailed description of the renormalization procedure within the present approach has been made in Ref. [7] and will be omitted here for brevity.

Using Eq. (10), one finds the known one-loop result for the perturbative field, which contains the dependence on the UV normalization point $\mu^{2}$ and IR cutoff $\lambda^{2}$ (e.g., Ref. [21]):

$$
W_{p t}^{(1)}\left(C_{\chi}\right)=-\frac{\alpha_{s}(\mu)}{2 \pi} C_{F} h(\chi) \ln \frac{\mu^{2}}{\lambda^{2}} .
$$

Therefore, in the leading order the kinematic dependence of the expression (7) is factorized into the function $h(\chi)$, which at large- $Q^{2}$ is approximated by

$$
h(\chi) \propto \ln \frac{Q^{2}}{m^{2}} .
$$

In this regime, the dependence of $W$ on the UV normalization scale $\mu$ (which can also be treated as an arbitrary factorization scale dividing the hard and soft subprocesses [24]) is governed by the renormalization group ( $\mathrm{RG})$ equation

$$
\left(\mu \frac{\partial}{\partial \mu}+\beta(g) \frac{\partial}{\partial g}\right) \frac{d \ln W\left(Q^{2}\right)}{d \ln Q^{2}}=-\Gamma_{\text {cusp }}\left[\alpha_{s}(\mu)\right],
$$

where $\Gamma_{\text {cusp }}\left(\alpha_{s}\right)$ is the universal cusp anomalous dimension evaluated in the perturbation theory. In Eq. (17), we take the logarithmic derivative in $Q^{2}$ in order to avoid problems with light-cone singularities at $m^{2}=0$ [24]. The solution of the $\mathrm{RG}$ equation leads to the evolution equation

$$
\frac{d \ln W\left(Q^{2}\right)}{d \ln Q^{2}}=-\int_{\lambda^{2}}^{\mu^{2}} \frac{d \xi}{2 \xi} \Gamma_{\text {cusp }}\left[\alpha_{s}(\xi)\right]+\frac{d W_{n p}\left(Q^{2}\right)}{d \ln Q^{2}},
$$

where the function $W_{n p}$ gives the initial condition at $\mu^{2}$ $=\lambda^{2}$ and has to be found by the nonperturbative methods $[25,26]$. Solving Eq. (18), we take the arbitrary upper bound for the squared momenta of soft gluons equal to the hard scale $\mu^{2}=Q^{2}$ and find

$$
\ln \frac{W\left(Q^{2}\right)}{W\left(Q_{0}^{2}\right)}=-\int_{Q_{0}^{2}}^{Q^{2}} \frac{d x}{x}\left[\int_{\lambda^{2}}^{x} \frac{d \xi}{2 \xi} \Gamma_{\text {cusp }}\left[\alpha_{s}(\xi)\right]-\frac{d W_{n p}(x)}{d \ln x}\right],
$$

which immediately leads to the conclusion that the leading large- $Q^{2}$ behavior of the quark form factor $F_{q}\left(Q^{2}\right)$ including all logarithmic corrections is controlled by the universal cusp anomalous dimension (17) and can be expressed in the following form (for comparison, see Ref. [24]):

$$
\begin{aligned}
F_{q}\left(Q^{2}\right)= & W\left(Q^{2}\right) \\
= & \exp \left[-\int_{Q_{0}^{2}}^{Q^{2}} \frac{d \xi}{2 \xi} \ln \frac{Q^{2}}{\xi} \Gamma_{\text {cusp }}\left[\alpha_{s}(\xi)\right]-\ln \frac{Q^{2}}{Q_{0}^{2}}\right. \\
& \left.\times \int_{\lambda^{2}}^{Q_{0}^{2}} \frac{d \xi}{2 \xi} \Gamma_{\text {cusp }}\left[\alpha_{s}(\xi)\right]+W_{n p}\left(Q^{2}\right)\right] W_{0},
\end{aligned}
$$

where $W_{0}=W\left(Q_{0}^{2}\right)$ contains both perturbative and nonperturbative contributions. From the one-loop result (15), the cusp anomalous dimension which satisfies the RG equation (17) in one-loop order is given by

$$
\Gamma_{\text {cusp }}^{(1)}\left[\alpha_{s}(\mu)\right]=\frac{\alpha_{s}(\mu)}{\pi} C_{F} .
$$

Substituting the anomalous dimension (21) in the one-loop approximation for the strong coupling into the Eq. (20), one finds

$$
\begin{aligned}
F_{q}^{(1)}\left(Q^{2}\right)= & \exp \left\{-\frac{2 C_{F}}{\beta_{0}}\left[\ln \frac{Q^{2}}{\Lambda^{2}} \ln \frac{\ln \left(Q^{2} / \Lambda^{2}\right)}{\ln \left(Q_{0}^{2} / \Lambda^{2}\right)}\right.\right. \\
& \left.\left.-\ln \frac{Q^{2}}{Q_{0}^{2}}\left(1-\ln \frac{\ln \left(Q_{0}^{2} / \Lambda^{2}\right)}{\ln \left(\lambda^{2} / \Lambda^{2}\right)}\right)\right]+W_{n p}\left(Q^{2}\right)\right\} \\
& \times F^{(1)}\left(Q_{0}^{2}\right),
\end{aligned}
$$

where $\Lambda$ is the QCD scale. The singularity in Eq. (22) originates from the region where the IR cutoff approaches $\Lambda$, i.e., where the coupling constant $\alpha_{s}$ increases, and then may have a nonperturbative nature.

\section{EFFECTS OF THE IR RENORMALONS}

In order to determine the structure of the nonperturbative function $W_{n p}$ in Eqs. (20), (22), it is instructive to study the corrections due to IR renormalons [9]. In the present situation, one can expect the corrections proportional to the powers of both scales $\mu^{2}$ and $\lambda^{2}$. However, taking into account the evolution in $\mu^{2}$ to the hard characteristic scale of the process $Q^{2}$ (19), we treat the power $\mu^{2}$ terms to be strongly suppressed, and focus on the power $\lambda^{2}$ corrections. To find them, let us consider the perturbative function $\Delta_{1}\left(\varepsilon, 0, \mu^{2} / \lambda^{2}\right)$ in the Eq. (10). The insertion of the fermion bubble 1-chain to the one-loop order expression (7) is equivalent to replacement of the frozen coupling constant $g^{2}$ by the running one $g^{2} \rightarrow g^{2}\left(k^{2}\right)=4 \pi \alpha_{s}\left(k^{2}\right)$ [25]:

$$
\widetilde{\Delta}_{1}\left(\varepsilon, 0, \mu^{2} / \lambda^{2}\right)=-4 \pi \mu^{2 \varepsilon} \int \frac{d^{n} k}{(2 \pi)^{n}} \alpha_{s}\left(k^{2}\right) \frac{\mathrm{e}^{i k z} \delta\left(z^{2}\right)}{k^{2}\left(k^{2}+\lambda^{2}\right)} .
$$

For the sake of convenience, we work here in Euclidean space. Using the integral representation for the one-loop running coupling $\alpha_{s}\left(k^{2}\right)=\int_{0}^{\infty} d \sigma\left(\Lambda^{2} / k^{2}\right)^{\sigma b}, b=\beta_{0} / 4 \pi$, we find

$$
\begin{aligned}
\widetilde{\Delta}_{1}\left(\varepsilon, 0, \mu^{2} / \lambda^{2}\right)= & -\frac{1}{\beta_{0}(1-\varepsilon)}\left(4 \pi \frac{\mu^{2}}{\lambda^{2}}\right)^{\varepsilon} \\
& \times \int_{0}^{\infty} d x \frac{\Gamma(1-x-\varepsilon) \Gamma(1+x+\varepsilon)}{(x+\varepsilon) \Gamma(1-\varepsilon)}\left(\frac{\Lambda^{2}}{\lambda^{2}}\right)^{x} .
\end{aligned}
$$

To define properly the integral on the right-hand side of Eq. (24), one needs to specify a prescription to go around the poles, which are at the points $\bar{x}_{n}=n, n \in \mathbb{N}$. Of course, the 
result of integration will depend on this prescription giving an ambiguity proportional to $\left(\Lambda^{2} / \lambda^{2}\right)^{n}$ for each pole. Then, the IR renormalons produce the power corrections to the one-loop perturbative result, which we assume to exponentiate with the latter $[25,26]$. Extracting from Eq. (24) the UV singular part in vicinity of the origin $x=0$, we divide the integration interval $[0, \infty]$ in two parts $[0, \delta]$ and $[\delta, \infty]$, where $\delta<1$. This procedure allows us to evaluate the ultraviolet and the renormalon-induced pieces separately. For the ultraviolet piece, we apply the expansion of the integrand in $\Delta_{1}$ in powers of small $x$ and replace the ratio of $\Gamma$ functions by $\exp \left(-\gamma_{E} \varepsilon\right)$ :

$$
\begin{aligned}
\widetilde{\Delta}_{1}^{\mathrm{UV}}\left(\varepsilon, 0, \mu^{2} / \lambda^{2}\right)= & -\frac{1}{\beta_{0}(1-\varepsilon)} \sum_{k, n=0}(-)^{n} \\
& \times \frac{\left(\ln 4 \pi-\gamma_{E}+\ln \frac{\mu^{2}}{\lambda^{2}}\right)^{k}}{k ! \varepsilon^{n-k+1}} \int_{0}^{\delta} d x x^{n}\left(\frac{\Lambda^{2}}{\lambda^{2}}\right)^{x},
\end{aligned}
$$

which after subtraction of the poles in the scheme becomes

$$
\begin{aligned}
\widetilde{\Delta}_{1}^{\mathrm{UV}}\left(0, \mu^{2} / \lambda^{2}\right)= & \frac{1}{\beta_{0}(1-\varepsilon)} \sum_{n=1}\left(\ln \frac{\mu^{2}}{\lambda^{2}}\right)^{n} \frac{(-)^{n}}{n !} \\
& \times \int_{0}^{\delta} d x x^{n-1}\left(\frac{\Lambda^{2}}{\lambda^{2}}\right)^{x} .
\end{aligned}
$$

In analogy with results of Ref. [27], this expression may be rewritten in a closed form as

$$
\begin{aligned}
& \tilde{\Delta}_{1}^{\mathrm{UV}}\left(0, \mu^{2} / \lambda^{2}\right) \\
& \quad=\frac{1}{\beta_{0}(1-\varepsilon)} \int_{0}^{\delta} \frac{d x}{x}\left[e^{-x \ln \left(\mu^{2} / \Lambda^{2}\right)}-e^{-x \ln \left(\lambda^{2} / \Lambda^{2}\right)}\right] .
\end{aligned}
$$

Substituting

$$
\frac{d W^{(1)}\left(Q^{2}\right)}{d \ln Q^{2}}=2 C_{F}(1-\varepsilon) \tilde{\Delta}_{1}^{\mathrm{UV}}\left(0, \mu^{2} / \lambda^{2}\right)
$$

into Eq. (17) one finds

$$
\begin{aligned}
& \left(\mu \frac{\partial}{\partial \mu}+\beta(g) \frac{\partial}{\partial g}\right) \frac{d \ln W^{(1)}\left(Q^{2}\right)}{d \ln Q^{2}} \\
& \quad=-\Gamma_{\text {cusp }}^{(1)}\left[\alpha_{s}(\mu)\right]\left(1-\exp \left[-\delta \frac{4 \pi}{\beta_{0} \alpha_{s}(\mu)}\right]\right) .
\end{aligned}
$$

The second exponent in the last equation yields the power suppressed terms $\left(\Lambda^{2} / Q^{2}\right)^{\delta}$ in large- $Q^{2}$ regime. In the leading logarithmic approximation (LLA) Eq. (28) is reduced to

$$
\frac{d W^{(1)}\left(Q^{2}\right)}{d \ln Q^{2}}=-\frac{2 C_{F}}{\beta_{0}}\left(\ln \frac{\ln \left(\mu^{2} / \Lambda^{2}\right)}{\ln \left(\lambda^{2} / \Lambda^{2}\right)}\right) .
$$

The last expression obviously satisfies the perturbative evolution equation (17).

The remaining integral in Eq. (24) over the interval $[\delta, \infty]$ is evaluated at $\varepsilon=0$ since there are no UV singularities. The resulting expression does not depend on the normalization point $\mu$, and thus it is determined by the IR region including nonperturbative effects. It contains the renormalon ambiguities due to different prescriptions in going around the poles $\bar{x}_{n}$ in the Borel plane which yields the power corrections to the quark form factor.

After the substitution $\mu^{2}=Q^{2}$ and integration over $d\left(\ln Q^{2}\right)$, we find in LLA [for comparison, see Eq. (22)]:

$$
\begin{aligned}
F_{q}^{\mathrm{ren}}\left(Q^{2}\right)= & \exp \left\{-\frac{2 C_{F}}{\beta_{0}}\left[\ln \frac{Q^{2}}{\Lambda^{2}} \ln \frac{\ln \left(Q^{2} / \Lambda^{2}\right)}{\ln \left(Q_{0}^{2} / \Lambda^{2}\right)}\right.\right. \\
& \left.\left.-\ln \frac{Q^{2}}{Q_{0}^{2}}\left(1-\ln \frac{\ln \left(Q_{0}^{2} / \Lambda^{2}\right)}{\ln \left(\lambda^{2} / \Lambda^{2}\right)}\right)\right]-\ln \frac{Q^{2}}{Q_{0}^{2}} \phi_{\mathrm{ren}}\left(\lambda^{2}, \Lambda^{2}\right)\right\} \\
& \times F^{\mathrm{ren}}\left(Q_{0}^{2}\right),
\end{aligned}
$$

where the function $\phi_{\text {ren }}\left(\lambda^{2}, \Lambda^{2}\right)=\sum_{k=0} \phi_{k}\left(\Lambda^{2} / \lambda^{2}\right)^{k}$ accumulates the effects of the IR renormalons, as well as the other nonperturbative information. The coefficients $\phi_{k}$ cannot be calculated in perturbation theory and can be treated as the minimal set of nonperturbative parameters. It is worth noting that the logarithmic $Q^{2}$ dependence of the renormalon induced corrections in the large- $Q^{2}$ regime is factorized, and thus the Eq. (31) reproduces exactly the structure of nonperturbative contributions found in the one-loop evolution equation (22) with respect to the large- $Q^{2}$ asymptotic behavior.

\section{LARGE- $Q^{2}$ BEHAVIOR OF THE INSTANTON INDUCED CONTRIBUTION}

Let us consider the instanton induced corrections to the perturbative result. The instanton field is given by

$\hat{A}_{\mu}(x ; \rho)=A_{\mu}^{a}(x ; \rho) \frac{\sigma^{a}}{2}=\frac{1}{g} \mathbf{R}^{a b} \sigma^{a} \eta^{ \pm b}{ }_{\mu \nu}\left(x-z_{0}\right)_{\nu} \varphi\left(x-z_{0} ; \rho\right)$,

where $\mathbf{R}^{a b}$ is the color orientation matrix $\left[a=1, \ldots,\left(N_{c}^{2}\right.\right.$ $-1), b=1,2,3], \sigma^{a}$ 's are the Pauli matrices, and ( \pm ) corresponds to the instanton, or anti-instanton. The averaging of the Wilson operator over the nonperturbative vacuum is reduced to the integration over the coordinate of the instanton center $z_{0}$, the color orientation and the instanton size $\rho$. The measure for the averaging over the instanton ensemble reads $d I=d \mathbf{R} d^{4} z_{0} d n(\rho)$, where $d \mathbf{R}$ refers to the averaging over color orientation and $d n(\rho)$ depends on the choice of the instanton size distribution. Taking into account Eq. (32), we write the Wilson integral (4), which defines the instanton induced contribution to the nonperturbative part in Eq. (20), in the single instanton approximation in the form

$$
W_{I}\left(C_{\chi}\right)=\frac{1}{N_{c}}\left\langle 0\left|\operatorname{Tr} \exp \left(i \sigma^{a} \phi^{a}\right)\right| 0\right\rangle,
$$


where

$$
\phi^{a}\left(z_{0}, \rho\right)=\mathbf{R}^{a b} \eta^{ \pm b} \int_{\mu \nu} d x_{\mu}\left(x-z_{0}\right)_{\nu} \varphi\left(x-z_{0} ; \rho\right) .
$$

We omit the path ordering operator $\mathcal{P}$ in Eq. (33) because the instanton field (32) is a hedgehog in color space, and so it locks the color orientation by space coordinates. Although in certain situations, the integrals of this type [Eq. (34)] can be evaluated explicitly [3], the calculation of the total integral (34) for a given contour requires an additional work, so we must restrict ourselves with the weak-field approximation. In contrast to our previous paper [7], here we use the cutoff $\lambda^{2}$ to regularize the IR divergences in the instanton case, while the UV divergences do not appear at all due to the finite instanton size. Then, in case of the instanton field, the LO contribution reads

$$
W_{I}^{(1)}\left(C_{\chi}\right)=2 h(\chi) \int d n(\rho) \Delta_{1}^{I}\left(0, \rho^{2} \lambda^{2}\right),
$$

where

$$
\Delta_{1}^{I}\left(0, \rho^{2} \lambda^{2}\right)=-\int \frac{d^{4} k}{(2 \pi)^{4}} e^{i k z} \delta\left(z^{2}\right)\left[2 \tilde{\varphi}^{\prime}\left(k^{2} ; \rho\right)\right]^{2} .
$$

Here, $\tilde{\varphi}\left(k^{2} ; \rho\right)$ is the Fourier transform of the instanton profile function $\varphi\left(z^{2} ; \rho\right)$ and $\tilde{\varphi}^{\prime}\left(k^{2} ; \rho\right)$ is it's derivative with respect to $k^{2}$. Note, that for the instanton calculations, it is necessary to map the scattering angle $\chi$ to the Euclidean space by the analytical continuation [28] $\chi \rightarrow i \gamma$, and perform the inverse transition to the Minkowski space-time in the final expressions in order to restore the $Q^{2}$ dependence. In the singular gauge, when the profile function is

$$
\varphi\left(z^{2}\right)=\frac{\rho^{2}}{z^{2}\left(z^{2}+\rho^{2}\right)},
$$

one gets

$$
\Delta_{1}^{I}\left(0, \rho^{2} \lambda^{2}\right)=\frac{\pi^{2} \rho^{4}}{4}\left[\ln \left(\rho^{2} \lambda^{2}\right) \Phi_{0}\left(\rho^{2} \lambda^{2}\right)+\Phi_{1}\left(\rho^{2} \lambda^{2}\right)\right],
$$

where

$$
\begin{aligned}
\Phi_{0}\left(\rho^{2} \lambda^{2}\right)= & \frac{1}{\rho^{4} \lambda^{4}} \int_{0}^{1} \frac{d z}{z(1-z)}\left[1+e^{\rho^{2} \lambda^{2}}-2 e^{z \cdot \rho^{2} \lambda^{2}}\right], \\
& \lim _{\lambda^{2} \rightarrow 0} \Phi_{0}\left(\rho^{2} \lambda^{2}\right)=1,
\end{aligned}
$$

and

$$
\begin{aligned}
\Phi_{1}\left(\rho^{2} \lambda^{2}\right)= & \sum_{n=1} \int_{0}^{1} d x d y d z \frac{\left\{-\rho^{2} \lambda^{2}[x z+y(1-z)]\right\}^{n}}{n ! n} \\
& \times e^{\rho^{2} \lambda^{2}[x z+y(1-z)]}, \underset{\lambda^{2} \rightarrow 0}{\lim } \Phi_{1}\left(\rho^{2} \lambda^{2}\right)=0
\end{aligned}
$$

are the IR-finite expressions. At high energy the instanton induced contribution is reduced to the form

$$
\begin{aligned}
\frac{d w_{I}\left(Q^{2}\right)}{d \ln Q^{2}} & =\frac{\pi^{2}}{2} \int d n(\rho) \rho^{4}\left[\ln \left(\rho^{2} \lambda^{2}\right) \Phi_{0}\left(\rho^{2} \lambda^{2}\right)+\Phi_{1}\left(\rho^{2} \lambda^{2}\right)\right] \\
& \equiv-B_{I}\left(\lambda^{2}\right)
\end{aligned}
$$

Here we used the exponentiation of the single-instanton result in a dilute instanton ensemble [7]:

$$
W_{I}=\exp \left(w_{I}\right)
$$

and took only the LO term of the weak-field expansion (7): $W^{(1)} \rightarrow w_{I}$.

In order to estimate the magnitude of the instanton induced effect we consider the standard distribution function [29] supplied with the exponential suppressing factor, which has been suggested in Ref. [30] (and discussed in Ref. [31] in the framework of constrained instanton model) in order to describe the lattice data [32]

$$
\begin{aligned}
d n(\rho)= & \frac{d \rho}{\rho^{5}} C_{N_{c}}\left(\frac{2 \pi}{\alpha_{s}\left(\mu_{r}\right)}\right)^{2 N_{c}} \exp \left(-\frac{2 \pi}{\alpha_{s}\left(\mu_{r}\right)}\right)\left(\rho \mu_{r}\right)^{\beta} \\
& \times \exp \left(-2 \pi \sigma \rho^{2}\right),
\end{aligned}
$$

where the constant $C_{N_{C}}$ is

$$
C_{N_{c}}=\frac{0.466 \mathrm{e}^{-1.679 N_{c}}}{\left(N_{c}-1\right) !\left(N_{c}-2\right) !} \approx 0.0015
$$

$\sigma$ is the string tension, $\beta=\beta_{0}+O\left[\alpha_{s}\left(\mu_{r}\right)\right]$, and $\mu_{r}$ is the normalization point [34]. Given the distribution (43) the main parameters of the instanton liquid model-the mean instanton size $\bar{\rho}$ and the instanton density $\bar{n}$-will read:

$$
\begin{aligned}
& \bar{\rho}=\frac{\Gamma(\beta / 2-3 / 2)}{\Gamma(\beta / 2-2)} \frac{1}{\sqrt{2 \pi \sigma}}, \\
& \bar{n}=\frac{C_{N_{c}} \Gamma(\beta / 2-2)}{2}\left(\frac{2 \pi}{\alpha_{S}\left(\bar{\rho}^{-1}\right)}\right)^{2 N_{c}}\left(\frac{\Lambda_{\mathrm{QCD}}}{\sqrt{2 \pi \sigma}}\right)^{\beta}(2 \pi \sigma)^{2} .
\end{aligned}
$$

In Eq. (46) we choose, for convenience, the normalization scale $\mu_{r}$ of order of the instanton inverse mean size $\bar{\rho}^{-1}$. Note, that these quantities correspond to the mean size $\rho_{0}$ and density $n_{0}$ of instantons used in the model [33], where the size distribution (43) is approximated by the delta function. $d n(\rho)=n_{0} \delta\left(\rho-\rho_{0}\right) d \rho$.

Thus, we find the leading instanton contribution (41) in the form 


$$
B_{I}=K \pi^{2} \bar{n} \bar{\rho}^{4} \ln \frac{2 \pi \sigma}{\lambda^{2}}\left[1+O\left(\frac{\lambda^{2}}{2 \pi \sigma}\right)\right]
$$

where

$$
K=\frac{\Gamma\left(\beta_{0} / 2\right)\left[\Gamma\left(\beta_{0} / 2-2\right)\right]^{3}}{2\left[\Gamma\left(\beta_{0} / 2-3 / 2\right)\right]^{4}} \approx 0.74,
$$

and we used the one loop expression for the running coupling constant

$$
\alpha_{s}\left(\bar{\rho}^{-1}\right)=-\frac{2 \pi}{\beta_{0} \ln \bar{\rho} \Lambda}, \quad \beta_{0}=\frac{11 N_{c}-2 n_{f}}{3} .
$$

The packing fraction $\pi^{2} \bar{n} \bar{\rho}^{4}$ characterizes diluteness of the instanton liquid and within the conventional picture its value is estimated to be 0.12 , if one takes the model parameters as (see Ref. [10])

$$
\bar{n} \approx 1 \mathrm{fm}^{-4}, \quad \bar{\rho} \approx 1 / 3 \mathrm{fm}, \quad \sigma \approx(0.44 \mathrm{GeV})^{2} .
$$

The leading contribution to the quark form factor at asymptotically large $Q^{2}$ is provided by the (perturbative) evolution governed by the cusp anomalous dimension (21). Thus, the instantons yield subleading effects to the large- $Q^{2}$ behavior accompanied by a numerically small factor

$$
B_{I} \approx 0.02 \text {, }
$$

as compared to the perturbative term $2 C_{F} / \beta_{0} \approx 0.24$.

Therefore, from Eqs. (41) and (31), we find the expression for the quark form factor at large $Q^{2}$ with the one-loop perturbative contribution and the nonperturbative contributions [the function $W_{n p}$ in Eq. (22)] which include both the IR renormalon and the instanton induced terms

$$
\begin{aligned}
F_{q}\left(Q^{2}\right)= & \exp \left\{-\frac{2 C_{F}}{\beta_{0}}\left[\ln \frac{Q^{2}}{\Lambda^{2}} \ln \frac{\ln \left(Q^{2} / \Lambda^{2}\right)}{\ln \left(Q_{0}^{2} / \Lambda^{2}\right)}\right.\right. \\
& \left.-\ln \frac{Q^{2}}{Q_{0}^{2}}\left(1-\ln \frac{\ln \left(Q_{0}^{2} / \Lambda^{2}\right)}{\ln \left(\lambda^{2} / \Lambda^{2}\right)}\right)\right] \\
& \left.-\ln \frac{Q^{2}}{Q_{0}^{2}}\left(B_{I}+\phi_{\text {ren }}\right)\right\} .
\end{aligned}
$$

It is clear that while the asymptotic (double-logarithmic) behavior is controlled by the perturbative cusp anomalous dimension, the leading nonperturbative corrections results in a finite renormalization of the next-to-leading (logarithmic) perturbative term. From the formal point of view, the evolution equation (20) describing the large- $Q^{2}$ asymptotic is valid even at the low scales $Q^{2} \sim 1 \mathrm{GeV}^{2}$, since the only condition of applicability of the Wilson integrals approach is $Q^{2} \gg \lambda^{2}, m^{2}$. However, in the low-energy domain the perturbative one-loop cusp anomalous dimension $\Gamma_{\text {cusp }}\left(\alpha_{s}\right)$ (21) should be supplemented by higher loop corrections, and thus the explicit formula (52) would include additional logarithmic terms. The relevance of the instanton induced part (41) in the low-energy domain calculated in the dilute gas ap- proximation can be questioned, and the additional consideration within more proper framework may help one to verify it. Indeed, the corrections to the single instanton approximation may be large at sufficiently low momenta (see for recent discussions, e.g., Ref. [35], and references therein). At the moment, we can say confidently that the evolution equation is valid at $Q^{2} \geqslant 1 \mathrm{GeV}^{2}$.

We have to comment that the weak field limit used in the instanton calculations may deviate from the exact result. Nevertheless, we expect that using of the instanton solution in the singular gauge, that concentrate the field at small distances, leads to the reasonable numerical estimate of the full effect. Thus, the resulting diminishing of the instanton contributions with respect to the perturbative result appears to be reasonable output. It should be emphasized that in the present paper, all the calculations have been performed analytically while the evaluation of the instanton contribution beyond the weak field approximation requires a numerical analysis, which will be the subject of a separate work. Moreover, the use of the singular gauge for the instanton solution allows us to prove the exponentiation theorem for the Wilson loop in the instanton field [7] which permits are to express the full instanton contribution as the exponent of the allorder single instanton result (42).

\section{CONCLUSION}

We analyzed the structure of the nonperturbative corrections to the quark form factor at large momentum transfer. In order to model the nonperturbative effects, we studied the quark scattering process in the background of the instanton vacuum. The instanton induced contribution to the color singlet quark form factor is calculated in the large momentum transfer regime. It was shown that the instanton induced corrections correspond to the leading term proportional to $\ln Q^{2}$. The magnitude of these corrections is determined by the small instanton liquid packing fraction parameter, and they can be treated as finite renormalization of the subleading perturbative part (52). In addition to this, the minimal set of the nonperturbative parameters is found considering infrared renormalon ambiguities of the perturbative series. Within this approach, it is shown that the leading large- $Q^{2}$ behavior of nonperturbative contributions should also be determined by the logarithmic term $\sim \ln \left(Q^{2}\right)$, what is consistent with the instanton analysis.

Let us emphasize that our results are quite sensitive to the prescription how to make the integration over instanton sizes finite. For example, if one used the sharp cutoff then the instanton would produce strongly suppressed power corrections such as $\propto(\Lambda / Q)^{\beta_{0}}$. However, we think that the distribution function (43) should be considered as more realistic, since it reflects more properly the structure of the instanton ensemble modeling the QCD vacuum. Indeed, this shape of distribution was recently advocated in Refs. [30,31] and supported by the lattice calculations [32].

Finally, we think that the instanton induced effects are more interesting for theoretical investigation and more important for phenomenology in the hadronic processes which possess two energy scales, such as the total center-of-mass 
energy $s$ (hard characteristic scale), and the squared momentum transfer $-t$ which is small compared to the latter: $-t$ $\ll s$. One of the most interesting examples of such processes is the parton-parton scattering and the soft Pomeron problem $[3,4,11]$. Another important situations where the nonperturbative (including instanton induced) effects can emerge are the transverse momentum distribution of vector bosons in the Drell-Yan process (see, e.g., Refs. [25,26]), and the phenomenon of saturation in deep-inelastic scattering (DIS) at small $x$ [36]. The explicit evaluation of the instanton effects in these processes will be the subject of our forthcoming study.

\section{ACKNOWLEDGMENTS}

We are grateful to A. P. Bakulev, N. I. Kochelev, O. V. Teryaev, and especially to S. V. Mikhailov for useful discussions. This work was partially supported by RFBR (Grant Nos. 03-02-17291, 02-02-81023, 02-02-16194, 01-0216431), the Heisenberg-Landau program (Grant No. HL2002-13), the Russian Federation President's Grant No. 1450-2003-2, and INTAS (Grant No. 00-00-366). One of the authors (I.Ch.) is grateful to the Theory group at DESY (Hamburg), and especially to F. Schrempp, for invitation, financial support, and numerous fruitful discussions.
[1] N. Andrei and D. Gross, Phys. Rev. D 18, 468 (1978); L. Baulieu, J. Ellis, M. Gaillard, and W. Zakrzewski, Phys. Lett. 77B, 290 (1978).

[2] I.I. Balitsky, Phys. Lett. B 273, 282 (1991); V.I. Zakharov, Nucl. Phys. B385, 452 (1992); I.I. Balitsky and V.M. Braun, Phys. Lett. B 314, 237 (1993).

[3] E. Shuryak and I. Zahed, Phys. Rev. D 62, 085014 (2000); M. Nowak, E. Shuryak, and I. Zahed, ibid. 64, 034008 (2001).

[4] D. Kharzeev, Y. Kovchegov, and E. Levin, Nucl. Phys. A690, 621 (2001).

[5] S. Moch, A. Ringwald, and F. Schrempp, Nucl. Phys. B507, 134 (1997).

[6] F. Schrempp and A. Utermann, Phys. Lett. B 543, 197 (2002).

[7] A. Dorokhov and I. Cherednikov, Phys. Rev. D 66, 074009 (2002).

[8] A. Ringwald and F. Schrempp, hep-ph/9411217.

[9] M. Beneke, Phys. Rep. 317, 1 (1999).

[10] T. Schäfer and E.V. Shuryak, Rev. Mod. Phys. 70, 323 (1998).

[11] G. Korchemsky, Phys. Lett. B 325, 459 (1994); I. Korchemskaya, Nucl. Phys. B490, 306 (1997).

[12] G. Korchemsky and A. Radyushkin, Phys. Lett. B 279, 359 (1992)

[13] Yu. Dokshitzer, D. Dyakonov, and S. Troyan, Phys. Rep. 58, 269 (1980).

[14] G. Parisi, Phys. Lett. 90B, 295 (1980); G. Curci and M. Greco, ibid. 92B, 175 (1980).

[15] G. Sterman, Nucl. Phys. B281, 310 (1987).

[16] L. Magnea and G. Sterman, Phys. Rev. D 42, 4222 (1990); L. Magnea, Nucl. Phys. B593, 269 (2001).

[17] R. Petronzio, S. Simula, and G. Ricco, Phys. Rev. D 67, 094004 (2003).

[18] J.M. Cornwall and G. Tiktopoulos, Phys. Rev. D 13, 3370 (1976); J. Frenkel and J.C. Taylor, Nucl. Phys. B116, 185 (1976); V.V. Belokurov and N.I. Usseyukina, Phys. Lett. 94B, 251 (1980).
[19] A. Bassetto, M. Ciafaloni, and G. Marchesini, Phys. Rep. 100, 201 (1983); O. Nachtmann, Ann. Phys. (N.Y.) 209, 436 (1991).

[20] Yu.M. Makeenko and A.A. Migdal, Phys. Lett. 88B, 135 (1979); Nucl. Phys. B188, 269 (1981); A. Polyakov, Gauge Fields and Strings (Harwood, Chur, 1987).

[21] G. Korchemsky and A. Radyushkin, Nucl. Phys. B283, 342 (1987).

[22] J.G.M. Gatheral, Phys. Lett. 133B, 90 (1983); J. Frenkel and J.C. Taylor, Nucl. Phys. B246, 231 (1984).

[23] R.A. Brandt, F. Neri, and M.-A. Sato, Phys. Rev. D 24, 879 (1981); R.A. Brandt, A. Gocksch, M.A. Sato, and F. Neri, ibid. 26, 3611 (1982); V. Dotsenko and S. Vergeles, Nucl. Phys. B169, 527 (1980); A. Polyakov, ibid. B164, 171 (1980); G. Korchemsky, Phys. Lett. B 217, 330 (1989).

[24] G. Korchemsky and A. Radyushkin, Sov. J. Nucl. Phys. 45, 910 (1987).

[25] G. Korchemsky and G. Sterman, Nucl. Phys. B437, 415 (1995).

[26] S. Tafat, J. High Energy Phys. 05, 004 (2001).

[27] S.V. Mikhailov, Phys. Lett. B 416, 421 (1998).

[28] E. Meggiolaro, Phys. Rev. D 53, 3835 (1996).

[29] G. 't Hooft, Phys. Rev. D 14, 3432 (1976).

[30] E. Shuryak, hep-ph/9909458.

[31] A.E. Dorokhov, S.V. Esaibegyan, and S.V. Mikhailov, Phys. Rev. D 56, 4062 (1997); A.E. Dorokhov, S.V. Esaibegyan, A.E. Maximov, and S.V. Mikhailov, Eur. Phys. J. C 13, 331 (2000).

[32] A. Hasenfratz and C. Nieter, Phys. Lett. B 439, 366 (1998).

[33] E. Shuryak, Nucl. Phys. B203, 93 (1982); B203, 116 (1982); B203, 140 (1982).

[34] T.R. Morris, D.A. Ross, and C.T. Sachrajda, Nucl. Phys. B255, 115 (1985).

[35] P. Faccioli, A. Schwenk, and E.V. Shuryak, Phys. Lett. B 549, 93 (2002).

[36] F. Schrempp and A. Utermann, hep-ph/0301177. 